\title{
Entropy, Similarity Measure, Inclusion Measure of Intuitionistic Fuzzy Sets and Their Relationships *
}

\author{
Qiansheng Zhang ${ }^{1 \dagger}$, Hongyan Xing ${ }^{2}$, Lihua $\mathrm{Wu}^{3}$ \\ ${ }^{1}$ School of Informatics, Guangdong University of Foreign Studies, \\ Guangzhou 510420, P.R. China \\ E-mail:zhqiansh01@126.com \\ ${ }^{2}$ Faculty of Applied Mathematics, Guangdong University of Technology, \\ Guangzhou 510006, P.R. China \\ E-mail:xhy@126.com \\ ${ }^{3}$ College of Informatics, South China Agricultural University, \\ Guangzhou 510642, P.R. China \\ E-mail:wulh@msn.com \\ Received 8 September 2011 \\ Accepted 9 April 2012
}

\begin{abstract}
In the present paper, we introduce a new axiomatic definition of the inclusion measure for intuitionistic fuzzy sets (IFSs, for short). The close relationships among entropy, similarity measure, and inclusion measure of IFSs are then discussed in detail. Also, we obtain some important theorems by which the entropy, similarity measure and inclusion measure of IFSs can be transformed into each other based on their axiomatic definitions. Moreover, some formulae for calculating the entropy, similarity measure and inclusion measure of IFSs are put forward. Finally, we compare the proposed new entropy, similarity and distance measures with the existing ones.
\end{abstract}

Keywords: Intuitionistic fuzzy set, Entropy, Similarity measure, Distance measure, Inclusion measure.

\section{Introduction}

To alleviate some drawbacks of Zadeh's fuzzy set (Rf.30), the concept of intuitionistic fuzzy set (IFS) was first introduced by Atanassov in 1986 (Rf.1) as an useful generalization of ordinary fuzzy set. It has been found to be more flexible and practical to deal with vagueness and uncertainty due to that intuitionistic fuzzy set can incorporate a hesi- tation in the membership degree, that is, assign to each element $u$ of $U$ a membership, a nonmembership and a hesitation degree. After the pioneering work of Atanassov, the IFS has received much attention from many researchers and has been used in more wide range of application fields. For example, Gerstenkorn (Rf.10) systematically investigated the correlation coefficients of IFSs. Coker (Rf.6) discussed the intuitionistic fuzzy topologi-

\footnotetext{
*This work is supported by the Humanities and Social Sciences Youth Foundation of Ministry of Education of China (No. 12YJCZH281). †Corresponding author: zhqiansh01@126.com (Q. S. Zhang)
} 
cal characteristic. Xu (Rf.25) studied the clustering technique of IFS. Li (Rf.15) proposed some multiattribute decision-making methods using IFSs. In addition, De (Rf.9) applied intuitionistic fuzzy set to medical diagnosis. Ciftcibasi (Rf.4) introduced Two-sided (intuitionistic) fuzzy reasoning approach. Cornelis (Rf.5) studied the implication in intuitionistic fuzzy theory.

As was well known, the entropy and similarity measure, and inclusion measure are three important issues in fuzzy set theory, which have been widely applied to pattern recognition, cluster analysis, image processing and decision making. With respect to intuitionistic fuzzy setting, the three concepts still play significant role in many deployed systems and application problems involving intuitionistic fuzzy sets.

The similarity measure of IFSs indicates the similar degree of two IFSs and plays an important role in pattern recognition, approximate reasoning and decision making. For instance, in (Rf.17) Szmidt presented a similarity measure of IFSs for supporting medical diagnostic reasoning. Later, Xu (Rf.26) proposed some similarity measures of IFs for multiple attribute decision making. Hung (Rf. 13) also presented a similarity measure of IFSs based on Hausdorff metric and applied it to pattern recognition.

The inclusion measure of fuzzy sets represents the degree to which a fuzzy set is contained in another fuzzy set. Sinha (Rf.19) first introduced an axiomatic definition of the inclusion measure of fuzzy sets. Later on, Cornelis (Rf.7) revised sinha's axiom, and proposed a inclusion for intuitionistic fuzzy set. Bustince (Rf.2) investigated the inclusion grade for interval-valued fuzzy sets. Kehagias (Rf.14) presented the concept of L-fuzzy valued inclusion measure, L-fuzzy valued similarity measure, and Lfuzzy distance.

Entropy, as a measure of fuzziness often used in the literature, describe the fuzziness degree or uncertain information of a fuzzy set and is first mentioned by Zadeh. It has received great attention recently. In 1972 (Rf.8) Deluca and Termini presented some axioms to describe the fuzziness degree of fuzzy set, with which a fuzzy entropy based on
Shannon's function was proposed. After that, many other researchers have studied the fuzzy entropy in different ways. Especially, Shang in (Rf.20) introduced another new fuzziness measure. For the information measure of intuitionistic fuzzy set, Szmidt (Rf.21) proposed an entropy for intuitionistic fuzzy set by employing a geometric interpretation of IFS. And Vlachos (Rf.22) studied the intuitionistic fuzzy information entropy and its application to pattern recognition. In 2010 Ye (Rf.29) also proposed two effective measures of intuitionistic fuzzy entropy. Moreover, Burillo (Rf.3) presented an entropy on interval-valued fuzzy sets and on intuitionistic fuzzy sets. And Vlachos (Rf.23) studied the subsethood and entropy of interval-valued fuzzy sets(IvFSs). Further Zeng (Rf.31) investigated the relationship between entropy and similarity measure of IvFSs from a different point of view. Additionally, we presented some new entropy formulae of vague set and interval-valued intuitionistic fuzzy sets, and their relations were then discussed in detail (Rf.32,33,34).

However, by now, although some entropy formulae, similarity measures and inclusion measures of IFSs have been introduced, and applied to many real life problems, there is little investigation on their relationships among them, which may be widely applied to many fields such as pattern recognition, cluster analysis, image segment. Therefore, it is worthwhile to focus on discovering the close relationships among entropy, similarity measure and inclusion measure for IFS. In section 2, we first summarize the axiomatic definitions of entropy, similarity measure and inclusion measure for IFS. Further, in section 3, some theorems reflecting the relationship among the entropy, the similarity measure, the inclusion measure are obtained, based on which some new formulae to evaluate entropy and similarity, inclusion measure of IFS are then put forward. The conclusion is given in the last section.

\section{Notations and Preliminaries}

Definition 2.1. Lattice $\left(L_{*}, \leqslant L_{*}, \vee, \wedge,,^{\prime}\right)$ is given by $L_{*}=\{(u, v) \in[0,1] \times[0,1] / u+v \leqslant 1\}$,
$\left(a_{1}, a_{2}\right) \leqslant L_{*}\left(b_{1}, b_{2}\right)$, iff $a_{1} \leqslant b_{1}$ and $a_{2} \geqslant b_{2}$,
$\left(a_{1}, a_{2}\right)=\left(b_{1}, b_{2}\right)$, iff $a_{1}=b_{1}, a_{2}=b_{2}$, 
$\left(a_{1}, a_{2}\right) \vee\left(b_{1}, b_{2}\right)=\left(\max \left\{a_{1}, b_{1}\right\}, \min \left\{a_{2}, b_{2}\right\}\right)$, $\left(a_{1}, a_{2}\right) \wedge\left(b_{1}, b_{2}\right)=\left(\min \left\{a_{1}, b_{1}\right\}, \max \left\{a_{2}, b_{2}\right\}\right)$, $\left(a_{1}, a_{2}\right)^{\prime}=\left(a_{2}, a_{1}\right), \quad \forall\left(\mathrm{a}_{1}, a_{2}\right),\left(b_{1}, b_{2}\right) \in L_{*} ;$ and $0_{L_{*}}=(0,1), 1_{L_{*}}=(1,0)$ are the smallest and greatest units of this lattice(Rf.5).

Notably, $\left(L_{*}, \leqslant_{L_{*}}\right)$ is a complete lattice since for any $M \subseteq L_{*}$ we know that

$\vee M=(\max \{x \in[0,1] \mid(\ni y \in[0,1])((x, y) \in$ $M)\}, \min \{y \in[0,1] \mid(\ni x \in[0,1])((x, y) \in M)\})$

$\in L_{*}$

$\wedge M=(\min \{x \in[0,1] \mid(\ni y \in[0,1])((x, y) \in$ $M)\}, \max \{y \in[0,1] \mid(\ni x \in[0,1])((x, y) \in M)\})$

$\in L_{*}$

and $\vee_{i}\left(a_{i 1}, a_{i 2}\right)=\left(\vee_{i} a_{i 1}, \wedge a_{i 2}\right)$,

$$
\wedge_{i}\left(a_{i 1}, a_{i 2}\right)=\left(\wedge_{i} a_{i 1}, \vee a_{i 2}\right), \quad \forall\left(\mathrm{a}_{i 1}, a_{i 2}\right) \in L_{*} .
$$

Definition 2.2 (Rf.1). An intuitionistic fuzzy set (IFS) $A$ in finite universe $X=\left\{x_{1}, x_{2}, \cdots, x_{n}\right\}$ is a mapping $A: X \rightarrow[0,1] \times[0,1]$, i.e., $A\left(x_{i}\right)=$ $\left(u_{A}\left(x_{i}\right), v_{A}\left(x_{i}\right)\right) \in L_{*}$ satisfying $0 \leqslant u_{A}\left(x_{i}\right)+v_{A}\left(x_{i}\right) \leqslant$ 1 for any $x_{i} \in X$, where $u_{A}\left(x_{i}\right), v_{A}\left(x_{i}\right)$ represent the degree of membership and nonmembership of that element $x_{i} \in X$ to the set $A$, respectively; and

$\Pi_{A}\left(x_{i}\right)=1-u_{A}\left(x_{i}\right)-v_{A}\left(x_{i}\right)$ represents the hesitation degree of the element $x_{i}$ to set $A$.

This definition favors IFS as they are readily seen to be $L_{*}$-fuzzy set w.r.t. the lattice $L_{*}$.

Throughout this paper, $X$ represents a finite universe unless specified, and we denote by $\mathscr{I} \mathscr{F}(X)$ the set of all intuitionistic fuzzy sets in finite universe $X, P(X)$ the power set of finite universe $X$.

Definition 2.3 (Rf.1). Let $A, B \in \mathscr{I} \mathscr{F}(X)$, the union, intersection, complement and inclusion as well as equality relation are defined as follows:

$A \cup B=\left\{\left\langle x_{i},\left(u_{A}\left(x_{i}\right) \vee u_{B}\left(x_{i}\right), \quad v_{A}\left(x_{i}\right) \wedge\right.\right.\right.$ $\left.\left.\left.v_{B}\left(x_{i}\right)\right)\right\rangle \mid x_{i} \in X\right\}$

$A \cap B=\left\{\left\langle x_{i},\left(u_{A}\left(x_{i}\right) \wedge u_{B}\left(x_{i}\right), \quad v_{A}\left(x_{i}\right) \vee\right.\right.\right.$ $\left.\left.\left.v_{B}\left(x_{i}\right)\right)\right\rangle \mid x_{i} \in X\right\}$;

$A^{\prime}=\left\{\left\langle x_{i},\left(v_{A}\left(x_{i}\right), u_{A}\left(x_{i}\right)\right)\right\rangle \mid x_{i} \in X\right\} ;$

$A \subseteq B, \quad$ iff $u_{A}\left(x_{i}\right) \leqslant u_{B}\left(x_{i}\right), \quad v_{A}\left(x_{i}\right) \geqslant$ $\left.v_{B}\left(x_{i}\right)\right), \forall x_{i} \in X$

$A=B, \quad$ iff $u_{A}\left(x_{i}\right)=u_{B}\left(x_{i}\right), \quad v_{A}\left(x_{i}\right)=$ $\left.v_{B}\left(x_{i}\right)\right), \forall x_{i} \in X$.

Hereafter, the notations $\vee, \wedge$ stand for max and min operations, respectively.

\section{Main axiomatic definitions}

Definition 3.1(Rf.21). A real function $E$ : $\mathscr{I} \mathscr{F}(X) \rightarrow[0,1]$ is named an entropy of IFSs on finite universe $X$, if $E$ satisfies all the following properties:

$\left(P_{1}\right) E(A)=0$ if $A$ is a crisp set, $\forall A \in P(X)$;

$\left(P_{2}\right) E(A)=1$ iff $u_{A}\left(x_{i}\right)=v_{A}\left(x_{i}\right), \forall x_{i} \in X$;

$\left(P_{3}\right) E(A) \leqslant E(B)$ if $A$ is less fuzzy than $B(A \ll B)$, which is defined as

$u_{A}\left(x_{i}\right) \leqslant u_{B}\left(x_{i}\right), v_{A}\left(x_{i}\right) \geqslant v_{B}\left(x_{i}\right)$, for $u_{B}\left(x_{i}\right) \leqslant v_{B}\left(x_{i}\right)$; $u_{A}\left(x_{i}\right) \geqslant u_{B}\left(x_{i}\right), v_{A}\left(x_{i}\right) \leqslant v_{B}\left(x_{i}\right)$, for $u_{B}\left(x_{i}\right) \geqslant v_{B}\left(x_{i}\right)$; $\left(P_{4}\right) E(A)=E\left(A^{\prime}\right)$.

Below, we display two entropy formulae of intuitionistic fuzzy set A, which fulfill all the above conditions $\left(P_{1}-P_{4}\right)$.

$$
\begin{gathered}
E_{1}(A)=1-\sum_{i=1}^{n}\left|u_{A}\left(x_{i}\right)-v_{A}\left(x_{i}\right)\right| / n \\
E_{2}(A)=1-\sqrt{\sum_{i=1}^{n}\left|u_{A}\left(x_{i}\right)-v_{A}\left(x_{i}\right)\right|^{2} / n}
\end{gathered}
$$

Definition 3.2(Rf.17). A real function $S: \mathscr{I} \mathscr{F}(X) \times$ $\mathscr{I} \mathscr{F}(X) \rightarrow[0,1]$ is named as the similarity measure of IFSs on universe $X$, if it satisfies properties:

$\left(S_{1}\right) S\left(A, A^{\prime}\right)=0$ if $A$ is a crisp set, $\forall A \in P(X)$;

$\left(S_{2}\right) S(A, B)=1$ iff $A=B$;

$\left(S_{3}\right) S(A, B)=S(B, A)$;

$\left(S_{4}\right)$ If $A \subseteq B \subseteq C$, then $S(A, C) \leqslant S(A, B)$ and $S(A, C) \leqslant S(B, C)$.

For instance, the following similarity measures of IFSs had been proposed in (Rf.25):

$S_{1}(A, B)=1-\frac{1}{2 n} \sum_{i=1}^{n}\left\{\left|u_{A}\left(x_{i}\right)-u_{B}\left(x_{i}\right)\right|+\left|v_{A}\left(x_{i}\right)-v_{B}\left(x_{i}\right)\right|\right\} ;$

$S_{2}(A, B)=1-\sqrt{\frac{1}{2 n} \sum_{i=1}^{n}\left\{\left|u_{A}\left(x_{i}\right)-u_{B}\left(x_{i}\right)\right|^{2}+\left|v_{A}\left(x_{i}\right)-v_{B}\left(x_{i}\right)\right|^{2}\right\}}$

Obviously, the above axiomatic definitions of entropy and similarity measure of IFS are extended from those of ordinary fuzzy set theory. Thus, if IFS $A$ in Def 3.1 becomes fuzzy set, then $E(A)$ reduces to the corresponding entropy of fuzzy set $A$; meanwhile, if IFSs $A, B$ in Def 3.2 become fuzzy sets, then $S(A, B)$ degenerates to the corresponding similarity measure of FSs. 
Also, in many real-life problems people usually need to numerically express the difference of two objects by means of the distance of the corresponding IFSs. For example, Grzegorzewski (Rf.11) presented a distance for IFSs based on Hausdorff metric. Szmidt and Kacprzyk (Rf.18) proposed some distance measures for IFSs and applied to pattern recognition. Wang (Rf.24) also applied some distance measures for IFSs to pattern recognition. Xu (Rf.27) systematically studied various of distance measures and similarity measures of IFSs. Thus, we also give the axiomatic definition of distance measure for IFSs below.

Definition 3.3 (Rf.18). A real function $d$ : $\mathscr{I} \mathscr{F}(X) \times \mathscr{I} \mathscr{F}(X) \rightarrow[0,1]$ is named as the distance measure of IFSs on universe $X$, if it satisfies the properties:

$\left(d_{1}\right) d\left(A, A^{\prime}\right)=1$ if $A$ is a crisp set, $\forall A \in P(X)$;

$\left(d_{2}\right) d(A, B)=0$ iff $A=B$;

$\left(d_{3}\right) d(A, B)=d(B, A)$;

$\left(d_{4}\right)$ If $A \subseteq B \subseteq C$, then $d(A, C) \geqslant d(A, B)$ and $d(A, C) \geqslant \bar{d}(B, C)$.

Note that the similarity measure can be deduced by distance measure as

For example:

$$
d(A, B)=1-S(A, B) .
$$

$d_{1}(A, B)=\frac{1}{2 n} \sum_{i=1}^{n}\left\{\left|u_{A}\left(x_{i}\right)-u_{B}\left(x_{i}\right)\right|+\left|v_{A}\left(x_{i}\right)-v_{B}\left(x_{i}\right)\right|\right\}$

$d_{2}(A, B)=\sqrt{\frac{1}{2 n} \sum_{i=1}^{n}\left|u_{A}\left(x_{i}\right)-u_{B}\left(x_{i}\right)\right|^{2}+\left|v_{A}\left(x_{i}\right)-v_{B}\left(x_{i}\right)\right|^{2}}$

Property 3.1. Assume $S_{i}(i=1,2)$ is the abovementioned similarity measure, then for any IFSs $A, B \in \mathscr{I} \mathscr{F}(X)$, we get

(1) $S_{i}(A, B)=S_{i}\left(A^{\prime}, B^{\prime}\right)$;

(2) $S_{i}(A, B)=S_{i}(A \cap B, A \bigcup B)$.

Property 3.2. Suppose $d_{i}(i=1,2)$ is the abovementioned distance measure, then for any IFSs $A, B \in \mathscr{I} \mathscr{F}(X)$, we obtain

(1) $d_{i}(A, B)=d_{i}\left(A^{\prime}, B^{\prime}\right)$;

(2) $d_{i}(A, A \cap B)=d_{i}(B, A \cup B)$;

(3) $d_{i}(A, A \cup B)=d_{i}(B, A \cap B)$.

Moreover, In 2000 (Rf.2) Bustince introduced an axiomatic definition for the inclusion grade of the interval-valued fuzzy sets and applied it to approximate reasoning. For intuitionistic fuzzy set, Cornelis (Rf.7) also proposed an inclusion measure. On account of the importance of inclusion measure in approximate reasoning, pattern recognition, we extend the subsethood (Rf.12,28] of FSs and introduce the axiomatic definition of inclusion measure for intuitionistic fuzzy sets based on the principle of set inclusion, which is different from Cornelis's work (Rf.7).

Definition 3.4. A real function $I: \mathscr{I} \mathscr{F}(X) \times$ $\mathscr{I} \mathscr{F}(X) \rightarrow[0,1]$ is named as the inclusion measure of IFSs on universe $X$, if it satisfies the following properties:

$\left(I_{1}\right) I(X, \phi)=0$;

(I $) I(A, B)=1$ iff $A \subseteq B$;

$\left(I_{3}\right)$ if $A \subseteq B \subseteq C$, then $I(C, A) \leqslant I(B, A)$ and $I(C, A) \leqslant I(C, B)$.

Obviously, if the IFSs $A, B$ become fuzzy sets, then $I(A, B)$ reduces to the subsethood of fuzzy sets. Now, we give two inclusion measures between IFSs $A, B$ as below.

$$
\begin{aligned}
& I_{1}(A, B)=1-\left[\frac { 1 } { 2 n } \sum _ { i = 1 } ^ { n } \left(\left|u_{A}\left(x_{i}\right)-u_{A \cap} B\left(x_{i}\right)\right|+\right.\right. \\
& \left.\left.\left|v_{A \cap B}\left(x_{i}\right)-v_{A}\left(x_{i}\right)\right|\right)\right] \text {; } \\
& I_{2}(A, B)=1- \\
& \sqrt{\frac{1}{2 n} \sum_{i=1}^{n}\left(\left|u_{A}\left(x_{i}\right)-u_{A \cap B}\left(x_{i}\right)\right|^{2}+\left|v_{A \cap B}\left(x_{i}\right)-v_{A}\left(x_{i}\right)\right|^{2}\right)} .
\end{aligned}
$$

\section{Relationships among entropy, similarity measure, inclusion measure of IFSs}

According to the above discussion, one can notice that the real functions of entropy, similarity measure and inclusion measure of IFSs are not unique. Therefore, in what follows, we will investigate on some important relationships among the similarity measure, the distance measure, the inclusion measure and the entropy of IFSs in detail. And then we will put forward some novel formulae to calculate the similarity measure, inclusion measure and entropy of IFSs.

Theorem 4.1. Assume $d$ is a distance measure of IFSs, for $A, B \in \mathscr{I} \mathscr{F}(X)$, then $S(A, B)=1-d(A, B)$ is a similarity measure of IFSs $A$ and $B$. 
Proof. The proof is straightforward from Def 3.2 and Definition 3.3.

Example 4.1. For $A, B \in \mathscr{I} \mathscr{F}(X)$, by utilizing the Hausdorff metric Grzegorzewski (Rf.11) suggested the following Hamming distance and Euclidean distance between them:

$d_{h}(A, B)=\frac{1}{n} \sum_{i=1}^{n}\left(\left|u_{A}\left(x_{i}\right)-u_{B}\left(x_{i}\right)\right| \vee\left|v_{A}\left(x_{i}\right)-v_{B}\left(x_{i}\right)\right|\right)$,

$d_{e}(A, B)=\sqrt{\frac{1}{n} \sum_{i=1}^{n}\left(\left|u_{A}\left(x_{i}\right)-u_{B}\left(x_{i}\right)\right|^{2} \vee\left|v_{A}\left(x_{i}\right)-v_{B}\left(x_{i}\right)\right|^{2}\right)}$

Then from Theorem 4.1 we get the corresponding similarity measures between IFSs.

$S_{h}(A, B)=1-\frac{1}{n} \sum_{i=1}^{n}\left(\left|u_{A}\left(x_{i}\right)-u_{B}\left(x_{i}\right)\right| \vee\left|v_{A}\left(x_{i}\right)-v_{B}\left(x_{i}\right)\right|\right)$,

$S_{e}(A, B)=1-\left[\frac{1}{n} \sum_{i=1}^{n}\left(\left|u_{A}\left(x_{i}\right)-u_{B}(x)\right|^{2} \vee\left|v_{A}\left(x_{i}\right)-v_{B}\left(x_{i}\right)\right|^{2}\right)\right]$

Example 4.2. For $A, B \in \mathscr{I} \mathscr{F}(X)$, Szmidt and Kacprzyk (Rf.17) also proposed the following normalized Hamming distance and Euclidean distance between them:

$$
\begin{gathered}
d_{5}(A, B)=\frac{1}{2 n} \sum_{i=1}^{n}\left(\left|u_{A}\left(x_{i}\right)-u_{B}\left(x_{i}\right)\right| \vee \mid v_{A}\left(x_{i}\right)-\right. \\
\left.v_{B}\left(x_{i}\right)|+| \Pi_{A}\left(x_{i}\right)-\Pi_{B}\left(x_{i}\right) \mid\right), \\
d_{6}(A, B)=\left\{\frac { 1 } { 2 n } \sum _ { i = 1 } ^ { n } \left(\left|u_{A}\left(x_{i}\right)-u_{B}\left(x_{i}\right)\right|^{2}+\mid v_{A}\left(x_{i}\right)-\right.\right. \\
\left.\left.\left.v_{B}\left(x_{i}\right)\right|^{2}+\left|\Pi_{A}\left(x_{i}\right)-\Pi_{B}\left(x_{i}\right)\right|^{2}\right)\right\}^{\frac{1}{2}} .
\end{gathered}
$$

Then from Theorem 4.1 we also get the corresponding similarity measures between IFSs.

$$
\begin{gathered}
S_{5}(A, B)=1-\frac{1}{2 n} \Sigma_{i=1}^{n}\left(\left|u_{A}\left(x_{i}\right)-u_{B}\left(x_{i}\right)\right| \vee \mid v_{A}\left(x_{i}\right)-\right. \\
\left.v_{B}\left(x_{i}\right)|+| \Pi_{A}\left(x_{i}\right)-\Pi_{B}\left(x_{i}\right) \mid\right), \\
S_{6}(A, B)=1-\left\{\frac { 1 } { 2 n } \Sigma _ { i = 1 } ^ { n } \left(\left|u_{A}\left(x_{i}\right)-u_{B}\left(x_{i}\right)\right|^{2}+\mid v_{A}\left(x_{i}\right)-\right.\right. \\
\left.\left.\left.v_{B}\left(x_{i}\right)\right|^{2}+\left|\Pi_{A}\left(x_{i}\right)-\Pi_{B}\left(x_{i}\right)\right|^{2}\right)\right\}^{\frac{1}{2}},
\end{gathered}
$$

where $\Pi_{A}\left(x_{i}\right), \Pi_{B}\left(x_{i}\right)$ denote the degree of hesitation of $x_{i}$ to IFS $A, B$, respectively.

Theorem 4.2. Assume $d$ is a distance measure of IFSs, for $A \in \mathscr{I} \mathscr{F}(X)$, if $f$ is a monotonic decreasing function from $[0,1]$ to $[0,1]$, then $E(A)=\frac{f\left(d\left(A, A^{\prime}\right)\right)-f(1)}{f(0)-f(1)}$ is an entropy of intuitionistic fuzzy set $A$.

Proof. We only need to prove that all the properties in Def 3.1 hold.

$\left(P_{1}\right)$ : If $A$ is a crisp set, i.e., $A \in P(X)$, and due to that $d$ is a similarity measure of IFSs, then from definition 3.3, we have $d\left(A, A^{\prime}\right)=1$.

Thus $E(A)=0$.

$\left(P_{2}\right)$ : If $u_{A}\left(x_{i}\right)=v_{A}\left(x_{i}\right), \forall x_{i} \in X$, then we know

$u_{A^{\prime}}\left(x_{i}\right)=v_{A}\left(x_{i}\right)=u_{A}\left(x_{i}\right), v_{A^{\prime}}\left(x_{i}\right)=u_{A}\left(x_{i}\right)=v_{A}\left(x_{i}\right)$.

Hence, $A^{\prime}=A$.

So, we get $d\left(A, A^{\prime}\right)=1$ by the Definition 3.3 of distance measure.

Then, $E(A)=\frac{f\left(d\left(A, A^{\prime}\right)\right)-f(1)}{f(0)-f(1)}=1$.

$\left(P_{3}\right)$ : If $A$ is less fuzzy than $B$, denoted by $A \ll B$, then we know,

when $u_{A}\left(x_{i}\right) \leqslant u_{B}\left(x_{i}\right), v_{A}\left(x_{i}\right) \geqslant v_{B}\left(x_{i}\right)$, for $u_{B}\left(x_{i}\right) \leqslant$ $v_{B}\left(x_{i}\right)$;

i.e., $u_{A}\left(x_{i}\right) \leqslant u_{B}\left(x_{i}\right) \leqslant v_{B}\left(x_{i}\right) \leqslant v_{A}\left(x_{i}\right)$,

so, $A \subseteq B \subseteq B^{\prime} \subseteq A^{\prime}$.

Therefore, by the definition 3.3 of distance measure of IFSs, we have

$$
d\left(A, A^{\prime}\right) \geqslant d\left(B, A^{\prime}\right) \geqslant d\left(B, B^{\prime}\right) .
$$

Similarly, when $u_{A}\left(x_{i}\right) \geqslant u_{B}\left(x_{i}\right), v_{A}\left(x_{i}\right) \leqslant v_{B}\left(x_{i}\right)$, for $u_{B}\left(x_{i}\right) \geqslant v_{B}\left(x_{i}\right)$;

i.e., $u_{A}\left(x_{i}\right) \geqslant u_{B}\left(x_{i}\right) \geqslant v_{B}\left(x_{i}\right) \geqslant v_{A}\left(x_{i}\right)$,

So, $A \supseteq B \supseteq B^{\prime} \supseteq A^{\prime}$.

Therefore, by the definition of distance measure of IFSs we get $d\left(A, A^{\prime}\right) \geqslant d\left(B, B^{\prime}\right)$.

Then,

$E(A)=\frac{f\left(d\left(A, A^{\prime}\right)\right)-f(1)}{f(0)-f(1)} \leqslant \frac{f\left(d\left(B, B^{\prime}\right)\right)-f(1)}{f(0)-f(1)}=E(B)$.

$\left(P_{4}\right)$ : From the definition of distance measure of IFSs, we know that

for any $A \in \mathscr{I} \mathscr{F}(X), d\left(A^{\prime}, A\right)=d\left(A, A^{\prime}\right)$ is trivial . Hence $E\left(A^{\prime}\right)=E(A)$.

Example 4.3. Let $A, B \in \mathscr{I} \mathscr{F}(X), \quad X=$ $\left\{x_{1}, x_{2}, \cdots, x_{n}\right\}$, according to the distance measures of IFSs proposed in (Rf.16), we have the following similarity measures of IFSs:

Normalized Hausdorff similarity measure induced by Hausdorff metric

$d_{h}^{*}(A, B)=\frac{1}{n} \sum_{i=1}^{n}\left[\left(\left|u_{A}\left(x_{i}\right)-u_{B}\left(x_{i}\right)\right| \vee\left|v_{A}\left(x_{i}\right)-v_{B}\left(x_{i}\right)\right|\right)\right]$,

Normalized Euclidean similarity measure induced by Euclidean distance

$d_{e}^{*}(A, B)=\left\{\frac{1}{2 n} \sum_{i=1}^{n}\left[\left(u_{A}\left(x_{i}\right)-u_{B}\left(x_{i}\right)\right)^{2}+\left(v_{A}\left(x_{i}\right)-v_{B}\left(x_{i}\right)\right)^{2}\right]\right\}^{\frac{1}{2}}$. 
Then from theorem 4.2 , by taking function $f(x)=$ $1-x$ we immediately obtain that

$$
\begin{gathered}
\frac{f\left(d\left(A, A^{\prime}\right)\right)-f(1)}{f(0)-f(1)}=1-\frac{1}{n} \sum_{i=1}^{n}\left|u_{A}\left(x_{i}\right)-v_{A}\left(x_{i}\right)\right|, \\
\frac{f\left(d\left(A, A^{\prime}\right)\right)-f(1)}{f(0)-f(1)}=1-\sqrt{\frac{1}{n} \sum_{i=1}^{n}\left(u_{A}\left(x_{i}\right)-v_{A}\left(x_{i}\right)\right)^{2},}
\end{gathered}
$$

are entropy formulae of IFS $A$, which correspond to $E_{1}(A), E_{2}(A)$, respectively.

Theorem 4.3. Suppose $S$ is a similarity measure of FSs, assume $A \in \mathscr{I} \mathscr{F}(X), X=\left\{x_{1}, x_{2}, \cdots, x_{n}\right\}$, then $S\left(\overline{u_{A}}, \overline{v_{A}}\right)$ is an entropy of IFS $A$, where $\overline{u_{A}}=1-u_{A}, \overline{v_{A}}=1-v_{A}$.

\section{Proof.}

$\left(P_{1}\right)$ : If $A \in P(X)$, then for any $x_{i} \in X$, $u_{A}\left(x_{i}\right)=1, v_{A}\left(x_{i}\right)=0$ or $u_{A}\left(x_{i}\right)=0, v_{A}\left(x_{i}\right)=1$; i.e., $v_{A}\left(x_{i}\right)=\left(u_{A}\left(x_{i}\right)\right)^{\prime}, \forall x_{i} \in X$.

Since $S$ is the similarity measure of fuzzy sets, we have $\quad S\left(\overline{u_{A}}, \overline{v_{A}}\right)=S\left(\overline{u_{A}}, u_{A}\right)=0$.

$\left(P_{2}\right)$ : From the definition of similarity measure, we immediately have

$S\left(\overline{u_{A}}, \overline{v_{A}}\right)=1 \Leftrightarrow \overline{u_{A}}=\overline{v_{A}} \Leftrightarrow 1-u_{A}\left(x_{i}\right)=1-$ $v_{A}\left(x_{i}\right) \Leftrightarrow u_{A}\left(x_{i}\right)=v_{A}\left(x_{i}\right), \forall x_{i} \in X$.

$\left(P_{3}\right)$ : Since $A$ is less fuzzy than $B$, then we know, when $u_{A}\left(x_{i}\right) \leqslant u_{B}\left(x_{i}\right), v_{A}\left(x_{i}\right) \geqslant v_{B}\left(x_{i}\right)$, for $u_{B}\left(x_{i}\right) \leqslant$ $v_{B}\left(x_{i}\right)$;

i.e., $u_{A}\left(x_{i}\right) \leqslant u_{B}\left(x_{i}\right) \leqslant v_{B}\left(x_{i}\right) \leqslant v_{A}\left(x_{i}\right)$,

So, $u_{A} \subseteq u_{B} \subseteq v_{B} \subseteq v_{A}$.

Known by the definition of similarity measure, we have $\quad S\left(u_{A}, v_{A}\right) \leqslant S\left(u_{A}, v_{B}\right) \leqslant S\left(u_{B}, v_{B}\right)$.

When $u_{A}\left(x_{i}\right) \geqslant u_{B}\left(x_{i}\right), v_{A}\left(x_{i}\right) \leqslant v_{B}\left(x_{i}\right)$, for $u_{B}\left(x_{i}\right) \geqslant$ $v_{B}\left(x_{i}\right)$;

then, $u_{A}\left(x_{i}\right) \geqslant u_{B}\left(x_{i}\right) \geqslant v_{B}\left(x_{i}\right) \geqslant v_{A}\left(x_{i}\right)$,

So, $\quad u_{A} \supseteq u_{B} \supseteq v_{B} \supseteq v_{A}$, and $\overline{u_{A}} \subseteq \overline{u_{B}} \subseteq \overline{v_{B}} \subseteq \overline{v_{A}}$.

By the definition of similarity measure, we get

$E(A)=S\left(\overline{u_{A}}, \overline{v_{A}}\right) \leqslant S\left(\overline{u_{B}}, \overline{v_{A}}\right) \leqslant S\left(\overline{u_{B}}, \overline{v_{B}}\right)=E(B)$.

$\left(P_{4}\right)$ : Since $A^{\prime}=\left\langle v_{A}, u_{A}\right\rangle$ and from the definition of similarity measure, it follows that

$E\left(A^{\prime}\right)=S\left(\overline{u_{A^{\prime}}}, \overline{v_{A^{\prime}}}\right)=S\left(\overline{v_{A}}, \overline{u_{A}}\right)=S\left(\overline{u_{A}}, \overline{v_{A}}\right)=E(A)$.

In the sequel, we can give some formulae to calculate the entropy of IFS based on some existing similarity measures of IFSs by Theorem 4.3.

Example 4.4. Suppose $A, B \in \mathscr{F}(X), X=$ $\left\{x_{1}, x_{2}, \cdots, x_{n}\right\}$, there are some widely used simi- larity measures of FSs as follows(Rf.26):

Hamming similarity measure induced by hamming distance measure:

$$
S_{1}^{*}(A, B)=1-\frac{1}{n} \sum_{i=1}^{n}\left(\left|A\left(x_{i}\right)-B\left(x_{i}\right)\right|\right) ;
$$

Euclidean similarity measure induced by Euclidean distance measure:

$$
S_{2}^{*}(A, B)=1-\sqrt{\frac{1}{n} \sum_{i=1}^{n}\left(\left|A\left(x_{i}\right)-B\left(x_{i}\right)\right|^{2}\right)} ;
$$

Similarity measure from the point of set-theoretic view:

$$
\begin{aligned}
S_{3}^{*}(A, B) & =\frac{\sum_{i=1}^{n}\left(\min \left\{A\left(x_{i}\right), B\left(x_{i}\right)\right\}\right)}{\sum_{i=1}^{n}\left(\max \left\{A\left(x_{i}\right), B\left(x_{i}\right)\right\}\right)} \\
S_{4}^{*}(A, B) & =\sum_{i=1}^{n} \frac{\min \left\{A\left(x_{i}\right), B\left(x_{i}\right)\right\}}{\max \left\{u_{A}\left(x_{i}\right), u_{B}\left(x_{i}\right)\right\}}
\end{aligned}
$$

Now, suppose $A=\left\langle u_{A}, v_{A}\right\rangle$ is an IFS. If we use $u_{A}, v_{A}$ to substitute for $A, B$ in the above similarity measures, respectively, then immediately get the corresponding entropies of $A$.

$$
\begin{gathered}
E_{1}^{*}(A)=1-\frac{1}{n} \sum_{i=1}^{n}\left|u_{A}\left(x_{i}\right)-v_{A}\left(x_{i}\right)\right|, \\
E_{2}^{*}(A)=1-\sqrt{\frac{1}{n} \sum_{i=1}^{n}\left|u_{A}\left(x_{i}\right)-v_{A}\left(x_{i}\right)\right|^{2},} \\
E_{3}^{*}(A)=\frac{\sum_{i=1}^{n}\left\{1-u_{A}\left(x_{i}\right) \vee v_{A}\left(x_{i}\right)\right\}}{\sum_{i=1}^{n}\left\{1-u_{A}\left(x_{i}\right) \wedge v_{A}\left(x_{i}\right)\right\}} \\
E_{4}^{*}(A)=\sum_{i=1}^{n} \frac{1-u_{A}\left(x_{i}\right) \vee v_{A}\left(x_{i}\right)}{1-u_{A}\left(x_{i}\right) \wedge v_{A}\left(x_{i}\right)},
\end{gathered}
$$

where $E_{1}^{*}(A), E_{2}^{*}(A)$ are the same as the abovementioned $E_{1}(A), E_{2}(A)$.

Theorem 4.4. Assume $A, B$ are two IFSs in $X=\left\{x_{1}, x_{2}, \cdots, x_{n}\right\}$ and for any $x_{i} \in X$, either $A\left(x_{i}\right) \subseteq B\left(x_{i}\right)$ or $B\left(x_{i}\right) \subseteq A\left(x_{i}\right)$, then for $i=1,2$,

$$
E_{i}^{*}(A)+E_{i}^{*}(B)=E_{i}^{*}(A \cup B)+E_{i}^{*}(A \cap B) \text {. }
$$

Proof. Here, we only prove that $E_{1}^{*}(A)$ satisfies the above equation.

$$
E_{1}^{*}(A)+E_{1}^{*}(B)=\left(1-\frac{1}{n} \sum_{i=1}^{n}\left|u_{A L}\left(x_{i}\right)-v_{A L}\left(x_{i}\right)\right|\right)
$$


$+\left(1-\frac{1}{n} \sum_{i=1}^{n}\left|u_{B}\left(x_{i}\right)-v_{B}\left(x_{i}\right)\right|\right)$

$E_{1}^{*}(A \cup B)=1-\frac{1}{n} \sum_{i=1}^{n}\left|u_{(A \cup B)}\left(x_{i}\right)-v_{(A \cup B)}\left(x_{i}\right)\right|$,

$E_{1}^{*}(A \cap B)=1-\frac{1}{n} \sum_{i=1}^{n}\left|u_{(A \cap B)}\left(x_{i}\right)-v_{(A \cap B)}\left(x_{i}\right)\right|$.

If $A \subseteq B$, i.e., $u_{A}\left(x_{i}\right) \leqslant u_{B}\left(x_{i}\right), v_{A}\left(x_{i}\right) \geqslant v_{B}\left(x_{i}\right)$, then we have

$$
\begin{aligned}
& E_{1}^{*}(A \cup B)=1-\frac{1}{n} \sum_{i=1}^{n}\left|u_{B}\left(x_{i}\right)-v_{B}\left(x_{i}\right)\right|, \\
& E_{1}^{*}(A \cap B)=1-\frac{1}{n} \sum_{i=1}^{n}\left|u_{A}\left(x_{i}\right)-v_{A}\left(x_{i}\right)\right|,
\end{aligned}
$$

So, $E_{1}(A \cup B)+E_{1}(A \cap B)=E_{1}(A)+E_{1}(B)$.

If $A \supseteq B$, i.e., $u_{A}\left(x_{i}\right) \geqslant u_{B}\left(x_{i}\right), v_{A}\left(x_{i}\right) \leqslant v_{B}\left(x_{i}\right)$, then we also have

$$
\begin{aligned}
& E_{1}^{*}(A \cup B)=1-\frac{1}{n} \sum_{i=1}^{n}\left|u_{A}\left(x_{i}\right)-v_{A}\left(x_{i}\right)\right|, \\
& E_{1}^{*}(A \cap B)=1-\frac{1}{n} \sum_{i=1}^{n}\left|u_{B}\left(x_{i}\right)-v_{B}\left(x_{i}\right)\right|,
\end{aligned}
$$

So, $E_{1}^{*}(A \cup B)+E_{1}^{*}(A \cap B)=E_{1}^{*}(A)+E_{1}^{*}(B)$.

Similarly, we can verify that $E_{2}^{*}$ satisfies the above equation, while $E_{3}^{*}, E_{4}^{*}$ do not fulfill.

Next, we further give a transform method of constructing similarity measure of IFSs based on the entropy of IFSs in the following.

Definition 4.1. Let $A, B$ be two IFSs in universe $X=\left\{x_{1}, x_{2}, \cdots, x_{n}\right\}$, assume $A\left(x_{i}\right)=\left\langle u_{A}\left(x_{i}\right), v_{A}\left(x_{i}\right)\right\rangle, \quad$ and $\quad B\left(x_{i}\right)=$ $\left\langle u_{B}\left(x_{i}\right), v_{B}\left(x_{i}\right)\right\rangle$,

we can define a new intuitionistic fuzzy set $\psi(A, B) \in \mathscr{I} \mathscr{F}(X)$ from $A, B$ as follows:

$u_{\psi(A, B)}\left(x_{i}\right)=\frac{1}{2}\left(1+\min \left\{\left|u_{A}\left(x_{i}\right)-u_{B}\left(x_{i}\right)\right|,\left|v_{A}\left(x_{i}\right)-v_{B}\left(x_{i}\right)\right|\right\}\right)$

$$
\text { In the similar way, we can get }
$$

$v_{\psi(A, B)}\left(x_{i}\right)=\frac{1}{2}\left(1-\max \left\{\left|u_{A}\left(x_{i}\right)-u_{B}\left(x_{i}\right)\right|,\left|v_{A}\left(x_{i}\right)-v_{B}\left(x_{i}\right)\right|\right\}\right) . \quad E(\psi(A, C)) \leqslant E(\psi(B, C))$.

Theorem 4.5. Let $E$ be an entropy of IFSs, for $A, B \in \mathscr{I} \mathscr{F}(X)$, then $E(\psi(A, B))$ is a similarity measure of IFSs $A$ and $B$.

Proof. We only need to prove that all the properties in Definition 3.2 hold.

$\left(S_{1}\right)$ : If $A \in P(X)$, then for any $x_{i} \in X$, we know $u_{A}\left(x_{i}\right)=1, v_{A}\left(x_{i}\right)=0$ or $u_{A}\left(x_{i}\right)=0, v_{A}\left(x_{i}\right)=1$.

Then

$u_{A^{\prime}}\left(x_{i}\right)=0, v_{A^{\prime}}\left(x_{i}\right)=1$ or $u_{A^{\prime}}\left(x_{i}\right)=1, v_{A^{\prime}}\left(x_{i}\right)=0$.

So, $u_{\psi\left(A, A^{\prime}\right)}\left(x_{i}\right)=\frac{1+\min \{1,1\}}{2}=1, \quad \forall x_{i} \in X$;

$$
v_{\psi\left(A, A^{\prime}\right)}\left(x_{i}\right)=\frac{1-\max \{1,1\}}{2}=0, \quad \forall x_{i} \in X ;
$$

$\psi\left(A, A^{\prime}\right)=\left\{\left\langle x_{i}, 1,0\right\rangle \mid x_{i} \in X\right\}$ is a crisp set in $X$.

From the axiomatic property $P_{1}$ of entropy $E$ in def-

inition 3.1, we have

$$
E\left(\psi\left(A, A^{\prime}\right)\right)=0 .
$$
know $u_{\psi(A, B)}\left(x_{i}\right) \geqslant v_{\psi(A, B)}\left(x_{i}\right)$.
$\left(S_{2}\right)$ : Known by the definition of entropy for IFS, we have

$$
\begin{aligned}
& E(\psi(A, B))=1 \\
\Leftrightarrow & u_{\psi(A, B)}=v_{\psi(A, B)} \\
\Leftrightarrow & \min \left\{\left|u_{A}\left(x_{i}\right)-u_{B}\left(x_{i}\right)\right|,\left|v_{A}\left(x_{i}\right)-v_{B}\left(x_{i}\right)\right|\right\}+ \\
& \max \left\{\left|u_{A}\left(x_{i}\right)-u_{B}\left(x_{i}\right)\right|,\left|v_{A}\left(x_{i}\right)-v_{B}\left(x_{i}\right)\right|\right\}=0 . \\
\Leftrightarrow & \left|u_{A}\left(x_{i}\right)-u_{B}\left(x_{i}\right)\right|+\left|v_{A}\left(x_{i}\right)-v_{B}\left(x_{i}\right)\right|=0 . \\
\Leftrightarrow & \left|u_{A}\left(x_{i}\right)-u_{B}\left(x_{i}\right)\right|=0, \text { and }\left|v_{A}\left(x_{i}\right)-v_{B}\left(x_{i}\right)\right|=0 . \\
\Leftrightarrow & u_{A}\left(x_{i}\right)=u_{B}\left(x_{i}\right), \quad v_{A}\left(x_{i}\right)=v_{B}\left(x_{i}\right), \quad \forall x_{i} \in X . \\
\Leftrightarrow & A=B .
\end{aligned}
$$

$\left(S_{3}\right)$ : From the definition of $\psi(A, B)$, we easily know that

$u_{\psi(A, B)}\left(x_{i}\right)=u_{\psi(B, A)}\left(x_{i}\right), v_{\psi(A, B)}\left(x_{i}\right)=v_{\psi(B, A)}\left(x_{i}\right) ;$ i.e., $\psi(A, B)=\psi(B, A)$.

Thus, $E(\psi(A, B))=E(\psi(B, A))$.

$\left(S_{4}\right)$ : If $A \subseteq B \subseteq C$, then for each $x_{i} \in X$, we know $u_{A}\left(x_{i}\right) \leqslant u_{B}\left(x_{i}\right) \leqslant u_{C}\left(x_{i}\right), \quad v_{A}\left(x_{i}\right) \geqslant v_{B}\left(x_{i}\right) \geqslant$ $v_{C}\left(x_{i}\right)$.

Hence, $\left|u_{A}\left(x_{i}\right)-u_{C}\left(x_{i}\right)\right| \geqslant\left|u_{A}\left(x_{i}\right)-u_{B}\left(x_{i}\right)\right|$,

$$
\left|v_{A}\left(x_{i}\right)-v_{C}\left(x_{i}\right)\right| \geqslant\left|v_{A}\left(x_{i}\right)-v_{B}\left(x_{i}\right)\right| \text {. }
$$

Thus, we get

$$
\begin{aligned}
& u_{\psi(A, C)}\left(x_{i}\right) \geqslant u_{\psi(A, B)}\left(x_{i}\right), \\
& v_{\psi(A, C)}\left(x_{i}\right) \leqslant v_{\psi(A, B)}\left(x_{i}\right), \quad \forall x_{i} \in X .
\end{aligned}
$$

Additionally, from the definition of $\psi(A, B)$, we

So, $\psi(A, C)$ is less fuzzy than $\psi(A, B)$.

Again known by the definition of entropy for IFS, we have

Therefore, $E(\psi(A, C)) \leqslant E(\psi(A, B)) \wedge E(\psi(B, C))$.

Corollary 4.1. Let $E$ be an entropy of IFS, assume $\psi(A, B) \in \mathscr{I} \mathscr{F}(X)$ is defined as above, then $E\left(\psi(A, B)^{\prime}\right)$ is also a similarity measure between IFSs $A$ and $B$. The proof is obvious (Omitted).

Definition 4.2. Let $A, B$ be two IFSs in universe $X=\left\{x_{1}, x_{2}, \cdots, x_{n}\right\}$. For any parameter $\alpha \in[1,+\infty)$, we define another new intuitionistic fuzzy set $\phi(A, B)$ from $A, B$ as

$$
\begin{aligned}
& u_{\phi(A, B)}\left(x_{i}\right)=\frac{1}{2}\left(1+\min \left\{\left|u_{A}\left(x_{i}\right)-u_{B}\left(x_{i}\right)\right|^{\alpha},\left|v_{A}\left(x_{i}\right)-v_{B}\left(x_{i}\right)\right|^{\alpha}\right\}\right) ; \\
& v_{\phi(A, B)}\left(x_{i}\right)=\frac{1}{2}\left(1-\max \left\{\left(\left|u_{A}\left(x_{i}\right)-u_{B}\left(x_{i}\right)\right|\right)^{\alpha},\left|v_{A}\left(x_{i}\right)-v_{B}\left(x_{i}\right)\right|^{\alpha}\right\}\right.
\end{aligned}
$$


Theorem 4.6. Let $E$ be an entropy of IFSs, then both $E(\phi(A, B))$ and $E\left(\phi(A, B)^{\prime}\right)$ are similarity measures of IFSs $A$ and $B$.

Proof. It can be similarly proved as Theorem 4.5 and Corollary 4.1 (Omitted).

Example 4.5. Assume $X=\left\{x_{1}, x_{2}, \cdots, x_{n}\right\}$ is a finite universe, and

$$
E(A)=1-\frac{1}{n} \sum_{i=1}^{n}\left|u_{A}\left(x_{i}\right)-v_{A}\left(x_{i}\right)\right|
$$

is an entropy of IFS $A$, then we can get the following similarity measure by theorem 4.5 .

$$
S(A, B)
$$

$$
\begin{aligned}
= & E(\psi(A, B)) \\
= & 1-\frac{1}{n} \sum_{i=1}^{n}\left(\left|u_{\psi(A, B)}\left(x_{i}\right)-v_{\psi(A, B)}\left(x_{i}\right)\right|\right) \\
= & 1-\frac{1}{n} \sum_{i=1}^{n}\left[\frac{1+\min \left\{\left|u_{A}\left(x_{i}\right)-u_{B}\left(x_{i}\right)\right|,\left|v_{A}\left(x_{i}\right)-v_{B}\left(x_{i}\right)\right|\right\}}{2}-\right. \\
& \left.\frac{1-\max \left\{\left|u_{A}\left(x_{i}\right)-u_{B}\left(x_{i}\right)\right|,\left|v_{A}\left(x_{i}\right)-v_{B}\left(x_{i}\right)\right|\right\}}{2}\right] \\
= & 1-\frac{1}{2 n} \sum_{i=1}^{n}\left(\left|u_{A}\left(x_{i}\right)-u_{B}\left(x_{i}\right)\right|+\left|v_{A}\left(x_{i}\right)-v_{B}\left(x_{i}\right)\right|\right) .
\end{aligned}
$$

In the sequel, we also put forward the other transform method of setting up entropy of IFS based on similarity measure of IFSs.

Let $A=\left\{\left\langle x_{i}, u_{A}\left(x_{i}\right), v_{A}\left(x_{i}\right)\right\rangle \mid x_{i} \in X\right\} \in \mathscr{I} \mathscr{F}(X)$ be an IFS in universe $X=\left\{x_{1}, x_{2}, \cdots, x_{n}\right\}$, we define the other two intuitionistic fuzzy sets $f(A), g(A)$ from given IFS $A$ as follows:

$$
\begin{aligned}
u_{f(A)}\left(x_{i}\right) & =\frac{1+\left|u_{A}\left(x_{i}\right)-v_{A}\left(x_{i}\right)\right|^{2}}{2}, \\
v_{f(A)}\left(x_{i}\right) & =\frac{1-\left|u_{A}\left(x_{i}\right)-v_{A}\left(x_{i}\right)\right|}{2} ; \\
\text { and } & \\
u_{g(A)}\left(x_{i}\right) & =\frac{1-\left|u_{A}\left(x_{i}\right)-v_{A}\left(x_{i}\right)\right|}{2}, \\
v_{g(A)}\left(x_{i}\right) & =\frac{1+\left|u_{A}\left(x_{i}\right)-v_{A}\left(x_{i}\right)\right|^{2}}{2} .
\end{aligned}
$$

Then, we immediately obtain the following theorem.

Theorem 4.7. Suppose $S$ is a similarity measure of IFSs, and $A$ is an IFS in the universe $X=\left\{x_{1}, x_{2}, \cdots, x_{n}\right\}$, then $S(f(A), g(A))$ is an entropy of IvIFS $A$.

\section{Proof.}

$\left(P_{1}\right)$ : If $A \in P(X)$, then for all $x_{i} \in X$,

$$
u_{A}\left(x_{i}\right)=1, v_{A}\left(x_{i}\right)=0 \text { or } u_{A}\left(x_{i}\right)=0, v_{A}\left(x_{i}\right)=1 \text {. }
$$

Further, from the definitions of $f(A), g(A)$, we have and

$$
u_{f(A)}\left(x_{i}\right)=\frac{1+1}{2}=1, \quad v_{f(A)}\left(x_{i}\right)=\frac{1-1}{2}=0 ;
$$

$$
u_{g(A)}\left(x_{i}\right)=\frac{1-1}{2}=0, \quad v_{g(A)}\left(x_{i}\right)=\frac{1+1}{2}=1 .
$$

So, $f(A)=X, g(A)=\emptyset$ are all crisp sets.

By the definition of similarity measure, we have $S(f(A), g(A))=S(X, \emptyset)=0$.

$\left(P_{2}\right)$ : Since $f(A), g(A) \in \mathscr{I} \mathscr{F}(X)$, and from the definition of similarity measure of IFSs it follows that

$$
S(f(A), g(A))=1
$$

$\Leftrightarrow f(A)=g(A)$

$\Leftrightarrow u_{f(A)}\left(x_{i}\right)=u_{g(A)}\left(x_{i}\right), \quad v_{f(A)}\left(x_{i}\right)=v_{g(A)}\left(x_{i}\right), \forall x_{i} \in$ $X$.

$\Leftrightarrow\left(\left|u_{A}\left(x_{i}\right)-v_{A}\left(x_{i}\right)\right|\right)\left(\left|u_{A}\left(x_{i}\right)-v_{A}\left(x_{i}\right)\right|+1\right)=$ $0, \forall x_{i} \in X$.

$\Leftrightarrow\left|u_{A}\left(x_{i}\right)-v_{A}\left(x_{i}\right)\right|=0, \quad \forall x_{i} \in X$.

$\Leftrightarrow u_{A}\left(x_{i}\right)=v_{A}\left(x_{i}\right), \quad \forall x_{i} \in X$.

$\left(P_{3}\right)$ : Suppose $A$ is less fuzzy than $B$, then we know, If $u_{A}\left(x_{i}\right) \leqslant u_{B}\left(x_{i}\right), v_{A}\left(x_{i}\right) \geqslant v_{B}\left(x_{i}\right), \quad$ for $u_{B}\left(x_{i}\right) \leqslant$ $v_{B}\left(x_{i}\right)$;

then $u_{A}\left(x_{i}\right) \leqslant u_{B}\left(x_{i}\right) \leqslant v_{B}\left(x_{i}\right) \leqslant v_{A}\left(x_{i}\right)$,

So, $\left|u_{A}\left(x_{i}\right)-v_{A}\left(x_{i}\right)\right| \geqslant\left|u_{B}\left(x_{i}\right)-v_{B}\left(x_{i}\right)\right|$,

Thus, $g(A) \subseteq g(B) \subseteq f(B) \subseteq f(A)$.

Known by the definition of similarity measure of IFSs, we have

$$
S(f(A), g(A)) \leqslant S(f(B), g(A)) \leqslant S(f(B), g(B)) .
$$

With the same reason, it follows that when $u_{A}\left(x_{i}\right) \geqslant u_{B}\left(x_{i}\right), \quad v_{A}\left(x_{i}\right) \leqslant v_{B}\left(x_{i}\right)$, for $u_{B}\left(x_{i}\right) \geqslant v_{B}\left(x_{i}\right)$;

then $u_{A}\left(x_{i}\right) \geqslant u_{B}\left(x_{i}\right) \geqslant v_{B}\left(x_{i}\right) \geqslant v_{A}\left(x_{i}\right)$.

So, $\left|u_{A}\left(x_{i}\right)-v_{A}\left(x_{i}\right)\right| \geqslant\left|u_{B}\left(x_{i}\right)-v_{B}\left(x_{i}\right)\right|$.

Thus, $g(A) \subseteq g(B) \subseteq f(B) \subseteq f(A)$.

Known by the definition of similarity measure of IFSs, we have

$$
\begin{aligned}
& E(A)=S(f(A), g(A)) \leqslant S(f(B), g(A)) \\
& \quad \leqslant S(f(B), g(B))=E(B) .
\end{aligned}
$$

Therefore, if $A$ is less fuzzy than $B$, then

$$
S(f(A), g(A)) \leqslant S(f(B), g(B)) \text {. }
$$

$\left(P_{4}\right)$ : From the definitions of $f(A), g(A)$, we know

$$
f(A)=f\left(A^{\prime}\right), \quad g(A)=g\left(A^{\prime}\right) .
$$

and due to the definition of similarity measure, it is obvious that

$E\left(A^{\prime}\right)=S\left(f\left(A^{\prime}\right), g\left(A^{\prime}\right)\right)=S(f(A), g(A))=E(A)$.

The proof is completed.

Corollary 4.2. Let $S$ be a similarity measure of IFSs, then $S\left((f(A))^{\prime},(g(A))^{\prime}\right)$ is an entropy of IFS $A$. The proof is similar to that of Theorem 4.7 (Omitted). 
Example 4.6. Assume $X=\left\{x_{1}, x_{2}, \cdots, x_{n}\right\}, A \in$ $\mathscr{I} \mathscr{F}(X)$, the Normalized Eculidean similarity measure is given as

$$
S(A, B)=1-\left\{\frac { 1 } { 2 n } \sum _ { i = 1 } ^ { n } \left[\left|u_{A}\left(x_{i}\right)-u_{B}\left(x_{i}\right)\right|^{2}+\right.\right.
$$$$
\left.\left.\left.\left|v_{A}\left(x_{i}\right)-v_{B}\left(x_{i}\right)\right|\right)^{2}\right]\right\}^{\frac{1}{2}} \text {, }
$$

from Theorem 4.7 we then know that

$$
\begin{aligned}
& S(f(A), g(A)) \\
= & 1-\left\{\frac { 1 } { 2 n } \sum _ { i = 1 } ^ { n } 2 ( \frac { 1 } { 2 } ) ^ { 2 } \left(\left|u_{A}\left(x_{i}\right)-v_{A}\left(x_{i}\right)\right|^{2}+\mid u_{A}\left(x_{i}\right)-\right.\right. \\
& \left.\left.v_{A}\left(x_{i}\right) \mid\right)^{2}\right\}^{\frac{1}{2}} \\
= & 1-\left\{\frac { 1 } { 4 n } \sum _ { i = 1 } ^ { n } \left(\left|u_{A}\left(x_{i}\right)-v_{A}\left(x_{i}\right)\right|^{4}+\mid u_{A}\left(x_{i}\right)-\right.\right. \\
& \left.\left.\left.v_{A}\left(x_{i}\right)\right|^{2}+2\left|u_{A}\left(x_{i}\right)-v_{A}\left(x_{i}\right)\right|^{3}\right)\right\}^{\frac{1}{2}}
\end{aligned}
$$

is an entropy of IFS $A$.

Example 4.7. Assume $X=[a, b], A \in \mathscr{I} \mathscr{F}(X)$, the Normalized hamming similarity measure is

$$
\begin{aligned}
& \quad S(A, B)=1-\frac{1}{2(b-a)} \int_{a}^{b}\left(\left|u_{A}(x)-u_{B}(x)\right|+\right. \\
& \left.\left|v_{A}(x)-v_{B}(x)\right|\right) d x, \\
& \text { then } \\
& \quad S(f(A), g(A)) \\
& =1-\frac{1}{4(b-a)} \int_{a}^{b} 4 \times \frac{1}{2}\left[\left|u_{A}(x)-v_{A}(x)\right|^{2}+\mid u_{A}(x)-\right. \\
& \left.\quad v_{A}(x) \mid\right] d x, \\
& =1-\frac{1}{2(b-a)} \int_{a}^{b}\left(\left|u_{A}(x)-v_{A}(x)\right|^{2}+\mid u_{A}(x)-\right. \\
& \left.\quad v_{A}(x) \mid\right) d x
\end{aligned}
$$

is also an entropy of IFS $A$.

Theorem 4.8. Let $E$ be an entropy of IFS, for any two IFSs $A, B \in \mathscr{I} \mathscr{F}(X)$, we define another intuitionistic fuzzy set $h(A, B)$ as follows:

$\mu_{h(A, B)}(x)=\frac{\left.1+\left[\left|\mu_{A}(x)-\mu_{A \cup B}(x)\right|+\left|v_{A}(x)-v_{A \cup B}(x)\right|\right) / 2\right]^{2}}{2}$,

$v_{h(A, B)}(x)=\frac{\left.1-\left[\left|\mu_{A}(x)-\mu_{A \cup B}(x)\right|+\left|v_{A}(x)-v_{A \cup B}(x)\right|\right) / 2\right]}{2}$,

then $E(h(A, B))$ is a inclusion measures of IFSs $A$ and $B$.

\section{Proof.}

$\left(I_{1}\right)$ If $A=X, B=\emptyset$, then

$h(A, B)(x)=\left(\mu_{h(A, B)}(x), v_{h(A, B)}(x)\right)=(1,0), \forall x \in X$. i.e., $h(A, B) \in \mathscr{P}(X)$,

Again by axiomatic property of entropy we know that $E(h(A, B))=0$.

(I $I_{2}$ If $A \subseteq B$, then $\mu_{h(A, B)}=v_{h(A, B)}=1 / 2$.

Thus, $E(h(A, B))=1$.

(I $I_{3}$ ) If $A \subseteq B \subseteq C$,

then $\mu_{h(C, A)} \geqslant \mu_{h(C, B)}, v_{h(C, A)} \leqslant v_{h(C, B)}$,

Again from $\mu_{h(C, B)} \geqslant v_{h(C, B)}$, it is easy to see that
$h(C, A) \ll h(C, B)$.

Thus, by the axiomatic definition of entropy we have $E(h(C, A)) \leqslant E(h(C, B))$.

Similarly, we can get $E(h(C, A)) \leqslant E(h(B, A))$.

Theorem 4.9. Assume that $d$ and $S$ are the distance and similarity measures of IFSs, then $I(A, B)=$ $S(A, A \cap B)=1-d(A, A \cap B)$ is an inclusion measure of IFS $A$ and $B$.

\section{Proof.}

$\left(I_{1}\right)$ Due to the definition of the similarity measure for IFSs, we get

$$
I(X, \phi)=S(X, X \cap \phi)=S(X, \phi)=0 .
$$

$\left(I_{2}\right)$ From the expression of $I(A, B)$, we have

$$
\begin{aligned}
I(A, B)=1 & \Leftrightarrow S(A, A \cap B)=1 \\
& \Leftrightarrow A=A \cap B \Leftrightarrow A \subseteq B .
\end{aligned}
$$

(I $\left.I_{3}\right)$ For $A \subseteq B \subseteq C$, then we get

$$
I(C, A)=S(C, C \cap A)=S(C, A),
$$

and $I(B, A)=S(B, B \cap A)=S(B, A)$.

Hence $I(C, A) \leqslant I(B, A)=S(B, A)$ by the definition of similarity measure for IFSs.

In the same manner, we can prove that

$I(C, A) \leqslant I(C, B)$.

Example 4.8. For $A, B \in \mathscr{I} \mathscr{F}(X)$, and we order distance measure as

$$
\begin{gathered}
d(A, B)=\frac{1}{2 n} \sum_{i=1}^{n}\left(\left|u_{A}\left(x_{i}\right)-u_{B}\left(x_{i}\right)\right|+\mid v_{A}\left(x_{i}\right)-\right. \\
\left.v_{B}\left(x_{i}\right)|+| \Pi_{A}\left(x_{i}\right)-\Pi_{B}\left(x_{i}\right) \mid\right),
\end{gathered}
$$

then we obtain the corresponding inclusion measure

$$
\begin{aligned}
& I(A, B)=S(A, A \cap B) \\
= & 1-\frac{1}{2 n} \sum_{i=1}^{n}\left(\left|u_{A}\left(x_{i}\right)-u_{A \cap B}\left(x_{i}\right)\right|+\mid v_{A}\left(x_{i}\right)-\right. \\
& \left.v_{A \cap B}\left(x_{i}\right)|+| \Pi_{A}\left(x_{i}\right)-\Pi_{A \cap B}\left(x_{i}\right) \mid\right) \\
= & 1-\frac{1}{2 n} \sum_{i=1}^{n}\left(\left|u_{A}\left(x_{i}\right)-u_{A}\left(x_{i}\right) \wedge u_{B}\left(x_{i}\right)\right|+\mid v_{A}\left(x_{i}\right) \vee\right. \\
& v_{B}\left(x_{i}\right)-v_{A}\left(x_{i}\right)|+| u_{A}\left(x_{i}\right) \wedge u_{B}\left(x_{i}\right)+v_{A}\left(x_{i}\right) \vee \\
& \left.v_{B}\left(x_{i}\right)-u_{A}\left(x_{i}\right)-v_{A}\left(x_{i}\right) \mid\right) .
\end{aligned}
$$

Theorem 4.10. Assume that $d$ and $S$ are the distance and similarity measures of IFSs, then $I(A, B)=S(B, A \cup B)=1-d(B, A \cup B)$ is the inclusion measure of IFS $A$ and $B$.

Proof. The proof is similar to that of Theorem 4.9, it is omitted.

Example 4.9 For $A, B \in \mathscr{I} \mathscr{F}(X)$, and we order distance measure as (Rf. 11,16)

$d(A, B)=\frac{1}{2 n} \sum_{i=1}^{n}\left(\left|u_{A}\left(x_{i}\right)-u_{B}\left(x_{i}\right)\right|+\left|v_{A}\left(x_{i}\right)-v_{B}\left(x_{i}\right)\right|\right)$, 
Then we obtain the corresponding inclusion measure

$$
\begin{gathered}
I(A, B)=S(B, A \cup B)=1-\frac{1}{2 n} \sum_{i=1}^{n}\left(\mid u_{B}\left(x_{i}\right)-u_{A}\left(x_{i}\right)\right. \\
\left.\vee u_{B}\left(x_{i}\right)|+| v_{A}\left(x_{i}\right) \wedge v_{B}\left(x_{i}\right)-v_{B}\left(x_{i}\right) \mid\right) .
\end{gathered}
$$

Theorem 4.11. Let $I$ be an inclusion measure of IFSs, for each $A \in \mathscr{I} \mathscr{F}(X), A^{\prime}$ be the complement of IFS $A$, then $E(A)=I\left(A^{\prime} \cup A, A^{\prime} \cap A\right)$ is an entropy of IFS $A$.

Proof. We need to prove that it satisfies all the axiomatic properties of entropy for IFS.

$\left(P_{1}\right)$ : If $A \in P(X)$, then $A^{\prime} \cup A=X, A^{\prime} \cap A=\emptyset$,

$$
\text { So, } I\left(A^{\prime} \cup A, A^{\prime} \cap A\right)=I(X, \phi) \text {. }
$$

Since $I$ is an inclusion measure, we have

$$
I\left(A^{\prime} \cup A, A^{\prime} \cap A\right)=I(X, \emptyset)=0 .
$$

$\left(P_{2}\right)$ : From property $\left(I_{2}\right)$ in the definition of inclusion measure, we immediately have

$$
\begin{aligned}
& I\left(A^{\prime} \cup A, A^{\prime} \cap A\right)=1 \Leftrightarrow A^{\prime} \cup A=A^{\prime} \cap A \\
\Leftrightarrow & A=A^{\prime} \Leftrightarrow u_{A}\left(x_{i}\right)=v_{A}\left(x_{i}\right), \quad \forall x_{i} \in X .
\end{aligned}
$$

$\left(P_{3}\right)$ : If $A$ is less fuzzy than $B$, then we deduce that

$$
A^{\prime} \cap A \subseteq B^{\prime} \cap B \subseteq B^{\prime} \cup B \subseteq A^{\prime} \cup A .
$$

With the property $\left(I_{3}\right)$ in definition 3.4 of inclusion measure, we get

$$
\begin{aligned}
E(A) & =I\left(A^{\prime} \cup A, A^{\prime} \cap A\right) \\
& \leqslant I\left(B^{\prime} \cup B, A^{\prime} \cap A\right) \\
& \leqslant I\left(B^{\prime} \cup B, B^{\prime} \cap B\right)=E(B) .
\end{aligned}
$$

$\left(P_{4}\right)$ : Since $A^{\prime}=\left\langle v_{A}, u_{A}\right\rangle, A^{\prime \prime}=A$, we have

$$
E\left(A^{\prime}\right)=I\left(A \cup A^{\prime}, A \cap A^{\prime}\right)=E(A) .
$$

Theorem 4.12. Let $I$ be an inclusion measure of IFSs, if a real function $S: \mathscr{I} \mathscr{F}(X) \times \mathscr{I} \mathscr{F}(X) \rightarrow$ $[0,1]$ satisfying $S(A, B)=I(A, B) \wedge I(B, A)$, then $\mathrm{S}$ is a similarity measure of IFSs.

\section{Proof.}

$\left(S_{1}\right) S\left(A, A^{\prime}\right)=I\left(A, A^{\prime}\right) \wedge I\left(A^{\prime}, A\right)=0$, if $A$ is a crisp set in $X$.

$$
\begin{gathered}
\left(S_{2}\right) \quad A=B \Leftrightarrow S(A, B)=I(A, B) \wedge I(B, A)=1 . \\
\left(S_{3}\right) S(A, B)=I(A, B) \wedge I(B, A) \\
\quad=I(B, A) \wedge I(A, B)=S(B, A) . \\
\left(S_{4}\right) \text { if } A \subseteq B \subseteq C, \text { then } I(A, B) \wedge I(B, A)=I(B, A), \\
S(A, C)=I(A, C) \wedge I(C, A)=I(C, A) \\
\leqslant I(B, A)=I(A, B) \wedge I(B, A)=S(A, B) .
\end{gathered}
$$

Similarly, we can get $S(A, C) \leqslant S(B, C)$.

\section{Conclusions}

In this paper, we summarize many entropy measures, similarity measures and inclusion measures, which have been applied to many real-world fields such as pattern recognition, clustering analysis and image processing. The close relationships among entropy, similarity measure, inclusion measure of IFSs are also investigated. Some new formulae to calculate entropy, similarity measure, and inclusion measure of IFS have been put forward. Also, we compare these measures with the existing ones and obtain some important theorems by which entropy, similarity measure, and inclusion measure of IFSs can be transformed into each other.

\section{Acknowledgments}

This work is supported by the Humanities and Social Sciences Youth Foundation of Ministry of Education of China (Nos. 12YJCZH281, 10YJCZH247, 11YJCZH086), the National Natural Science Foundation (Nos. 60974019, 61070061, 60964005), and the Guangdong Province High-level Talents Project.

\section{References}

1. K. Atanassov, "Intuitionistic fuzzy sets," Fuzzy Sets and Systems , 20, 87-96 (1986).

2. H. Bustince, "Indicator of inclusion grade for intervalvalued fuzzy sets: Application to approximate reasoning based on interval-valued fuzzy sets," International Journal of Approximate Reasoning , 23, 137209 (2000).

3. P. Burillo, and H. Bustince, "Entropy on intuitionistic fuzzy sets and on interval-valued fuzzy sets," Fuzzy Sets and Systems , 78, 305-316 (1996).

4. T. Ciftcibasi, and D. Altunay, "Two-sided (intuitionistic) fuzzy reasoning," IEEE Transactions on Systems, Man and Cybernetics, , 28, 662-677 (1998).

5. C. Cornelis, and G. Deschrijver, "Implication in intuitionistic fuzzy and interval-valued fuzzy set theory: construction, classification, application," International Journal of Approximate Reasoning , 35, 55-95 (2004).

6. D. Coker, "An introduction to intuitionistic fuzzy topological spaces," Fuzzy Sets and Systems, ,88, 8189 (1997).

7. C. Cornelis, "Inclusion measures in intuitionistic fuzzy set theory, Symbolic and Quantitative Ap- 
proaches to Reasoning with Uncertainty, " Lecture Notes in Computer Science, 2711 , 345-356 (2004).

8. A. DeLuca, and S. Termini, "A definition of nonprobabilistic entropy in the setting of fuzzy sets theory," Information and Control, 20, 301-312 (1972).

9. S. K. De, R. Biswas, and A. R. Roy, "An application of intuitionistic fuzzy sets in medical diagnosis, "Fuzzy Sets and Systems, 117, 209-213 (2001).

10. T. Gerstenkorn, and J. Mako, "Correlation of intuitionistic fuzzy sets," Fuzzy Sets and Systems, 44, 3943 (1991).

11. P. Grzegorzewski, "Distances between intuitionistic fuzzy sets and/or interval-valued fuzzy sets based on the Hausdorff metric," Fuzzy Sets and Systems, 148, 319-328 (2004)

12. M. Galar, H. Bustince, J. Fernandez, J. Sanz, and G. Beliakov, "Fuzzy entropy from weak fuzzy subsethood measures, Neural network world," International Journal of Approximate Reasoning , 20, 139158 (2010)

13. W. L. Hung, "Similarity measures of intuitionistic fuzzy sets based on Hausdorff distance," Pattern Recognition Letters, 25, 1603-1611 (2004).

14. A. Kehagias, "L-fuzzy valued inclusion measure, Lfuzzy similarity and L-fuzzy distance," Fuzzy Sets and Systems, 136, 313-332 (2003).

15. D. F. Li, "Multiattribute decision making models and methods using intuitionistic fuzzy sets," Journal of Computer and System Sciences, 70, 73-85 (2005).

16. J. H. Park, K.M. Lim, and J.S. Park, "Distances between interval-valued intuitionistic fuzzy sets,"Journal of Physics: Conference Series , 96, 1-8 (2008).

17. E. Szmidt, and J. Kacprzyk, "A similarity measure for intuitionistic fuzzy sets and its application in supporting medical diagnostic reasoning," Lecture Notes in Computer Science, 3070, 388-393 (2004).

18. E. Szmidt, and J. Kacprzyk, " Distances between intuitionistic fuzzy sets, " Fuzzy Sets and Systems, 114 505-518 (2000).

19. D. Sinha, and E. R. Dougherty, "Fuzzification of set inclusion: theory and applications," Fuzzy sets and systems, 55, 15-42 (1993).

20. X. G. Shang, and W. S. Jiang, “ A note on fuzzy information measures," Pattern Recognition Letters, 18 ,
425-432 (1997).

21. E. Szmidt, "Entropy for intuitionistic fuzzy sets," Fuzzy sets and systems, 118, 467-477 (2001).

22. I. K. Vlachos, " Intuitionistic fuzzy informationApplications to pattern recognition, " Pattern Recognition Letters , 28, 197-206. (2007).

23. I. K. Vlachos, "Subsethood, entropy, and cardinality for interval-valued fuzzy sets-An algebraic derivation," Fuzzy sets and systems, 158, 1384-1396 (2007).

24. W. Wang, and X. Xin, "Distance measure between intuitionistic fuzzy sets, " Pattern Recognition Letters 26, 2063-2069 (2005).

25. Z.S. Xu, J. Chen, and J.J. Wu, " Clustering algorithm for intuitionistic fuzzy sets," Information Sciences, 178, 3775-3790 (2008).

26. Z. S. Xu, "Some similarity measures of intuitionistic fuzzy sets and their applications to multiple attribute decision making," Fuzzy Optimization and Decision Making , 6, 109-121 (2007).

27. Z. S. Xu, "An overview of distance and similarity measures of intuitionistic fuzzy sets," International Journal of Uncertainty, Fuzziness and KnowledgeBased Systems , 16, 529-555 (2008).

28. V. R. Young, “Fuzzy subsethood, " Fuzzy Sets and Systems , 77, 371-384 (1996).

29. J. Ye, "Two effective measures of intuitionistic fuzzy entropy," Computing , 87, 55-62 (2010).

30. L. A. Zadeh, "Fuzzy sets, " Information and control, 8, 338-353 (1965).

31. W. Zeng, " Relationship between similarity measure and entropy of interval valued fuzzy sets, " Fuzzy Sets and Systems, 157, 1477-1484 (2006)

32. Q. S. Zhang, and S. Y. Jiang, " A note on information entropy measures for vague sets and its applications, "Information Sciences , 178, 4184-4191 (2008).

33. Q. S. Zhang, S. Y. Jiang, B.G.Jia, and S.H. Luo, "Some information entropy measures for intervalvalued intuitionistic fuzzy sets," Information Sciences , 180, 5130-5145 (2010).

34. Q. S. Zhang, and S. Y. Jiang, “ Relationships between entropy and similarity measure of interval-valued intuitionistic fuzzy sets," International Journal of Intelligent Systems , 25, 1121-1140 (2010). 